\title{
Fibrosing Alopecia in a Pattern Distribution (FAPD) in 16 African-Descent and Hispanic Female Patients: A Challenging Diagnosis
}

\author{
Marcelo de Souza Teixeira ${ }^{a}$ Maria Fernanda Reis Gavazzoni Dias ${ }^{a}$ \\ Ralph M. Trüeb ${ }^{b}$ Mayra Carrijo Rochael ${ }^{c}$ Enoi Aparecida Guedes Vilar ${ }^{c}$ \\ a Department of Dermatology, Hospital Universitário Antonia Pedro, Universidade Federal Fluminense, Niterói, \\ Brazil; ${ }^{b}$ Center for Dermatology and Hair Diseases Professor Trüeb, Wallisellen, Switzerland; ' Division of \\ Dermatopathology, Department of Dermatology, Universidade Federal Fluminense, Niterói, Brazil
}

\section{Keywords}

Female alopecia · Androgenetic alopecia - Cicatricial alopecia $\cdot$ Fibrosing alopecia in a pattern distribution

\begin{abstract}
Background: Fibrosing alopecia in a pattern distribution (FAPD) has only been described in Caucasian patients, and it is not clear whether it can develop in dark-skin ethnicities. Materials and Methods: Sixteen Brazilian female patients, 12 of African descent and 4 Hispanic, with progressive scarring alopecia in a pattern distribution were analyzed. $\boldsymbol{R} \boldsymbol{e}$ sults: Dermatoscopic features showed perifollicular erythema and scaling (14/16), hair fiber diameter diversity (16/16), loss of follicular ostia (16/16), and follicular keratosis (3/16). Late stages showed a honeycomb pigmented network (12/16), a hyperpigmented perifollicular halo (12/16), and small white patches (12/16). Histopathological features showed lichenoid perifollicular infiltrate (14/16), follicular miniaturization (16/16), concentric fibrosis (16/16), perifollicular lymphocytic infiltrate (16/16), and vellus hair involvement (10/16). Premature desquamation of the inner root sheath was found in 11 patients. Conclusions: The concomitant findings of cicatricial pattern hair loss (with or without
\end{abstract}

(c) 2019 S. Karger AG, Basel

E-Mail karger@karger.com www.karger.com/sad the recess of the front hair line), hair fiber diversity, perifollicular erythema and scaling, a whitish perifollicular halo, and histological findings of androgenetic alopecia, with vacuolar interface alteration of the upper portion of the follicular epithelium, are the main key features to suggest the diagnosis of FAPD. FAPD is a possible diagnosis in patients of color with cicatricial pattern hair loss. Clinical, dermatoscopic, and histopathological examination allow a proper final differential diagnosis.

c) 2019 S. Karger AG, Basel

\section{Introduction}

Fibrosing alopecia in a pattern distribution (FAPD) is a form of cicatricial pattern alopecia described in 2000 by Zinkernagel and Trüeb [1] as a possible variant of the lymphocytic primary cicatricial alopecia group [1]. The hallmark of this type of alopecia is the combination of clinical and histopathological features of lichen planopilaris and androgenetic alopecia (AGA) [1-6]. Since the first description of FAPD only included Caucasians, the study of this condition in patients of color needs to be revisited. We present 12 African-descent and 4 Hispanic 
Brazilian female patients with progressive scarring alopecia in a male or female pattern distribution and analyze the clinical, dermatoscopic, and histopathologic features of the condition $[1,2]$.

\section{Patients and Methods}

We analyzed 12 African-descent and 4 Hispanic female patients aged between 29 and 75 years with cicatrical pattern alopecia, as described by Olsen [5], complaining of gradual decrease in hair density and without any history of acute hair loss. All patients were under evaluation at the Hospital Universitário Antônio Pedro, Niterói, Brazil, between 2016 and 2017, and were biopsied in two different areas of the scalp (frontal and parietal areas) with a

Table 1. Dermatoscopic features

\begin{tabular}{lrc}
\hline Perifollicular erythema and scaling & $14 / 16$ & 87.5 \\
Loss of follicular ostia & $16 / 16$ & 100 \\
White perifollicular halo & $9 / 16$ & 56.25 \\
Hyperpigmented perifollicular halo & $12 / 16$ & 75 \\
Keratotic follicular plugs & $3 / 16$ & 18.75 \\
Scattered white small patches & $12 / 16$ & 75 \\
Hair diameter diversity & $16 / 16$ & 100 \\
Honeycomb pigmented network & $12 / 16$ & 75 \\
\hline
\end{tabular}

Values are presented as $n / N$ and $\%$. 4-mm punch each. Biopsies were dermoscopy guided. The optimal site for biopsy presented perifollicular erythema and scaling, keratotic follicular plugging, and/or a peripilar gray-whitish halo $[1,7]$. Specimens were transvertionally sectioned two times to study three different levels: the first level at $1 \mathrm{~mm}$ below the epidermis (infundibulum), the second level at $1 \mathrm{~mm}$ below the first one (isthmus), and a third level (suprabulbar) $[2,8]$.

\section{Results}

All patients presented clinical signs of cicatricial alopecia in an androgenetic distribution. Ten patients presented female pattern hair loss distribution, 4 patients had male pattern hair loss distribution, and 2 patients had diffuse hair thinning associated with recession of the frontotemporal hairline.

Clinical, dermatoscopic, and histopathological features are exhibited in Figure 1 and Tables 1 and 2.

\section{Discussion}

FAPD in Caucasians was first described by Zinkernagel and Trüeb [1] as a type of cicatricial pattern alopecia with histopathological features consistent with AGA, lichen planopilaris, and frontal fibrosing alopecia (FFA)

Table 2. Histopathological features of the scalp biopsy samples

\begin{tabular}{|c|c|c|c|c|c|c|c|c|c|}
\hline Patient & $\begin{array}{l}\text { Lichenoid infiltrate } \\
\text { (interface dermatitis at } \\
\text { isthmus level) }\end{array}$ & $\begin{array}{l}\text { Concentric } \\
\text { fibrosis }\end{array}$ & $\begin{array}{l}\text { Fibrous } \\
\text { tracts }\end{array}$ & PDIRS & AGA & $\begin{array}{l}\text { Perivascular } \\
\text { lymphocytic } \\
\text { infiltrate }\end{array}$ & $\begin{array}{l}\text { Follicular } \\
\text { keratinocyte } \\
\text { apoptosis }\end{array}$ & $\begin{array}{l}\text { Vellus hair } \\
\text { involvement }\end{array}$ & Polytrichia \\
\hline 1 & yes & yes & yes & no & yes & yes & no & yes & no \\
\hline 2 & yes & yes & yes & no & yes & yes & yes & yes & no \\
\hline 3 & yes & yes & yes & yes & yes & yes & no & no & no \\
\hline 4 & yes & yes & yes & yes & yes & yes & yes & yes & no \\
\hline 5 & yes & yes & yes & yes & yes & yes & no & no & no \\
\hline 6 & yes & yes & no & no & yes & yes & no & yes & no \\
\hline 7 & yes & yes & yes & yes & yes & yes & yes & yes & no \\
\hline 8 & yes & yes & yes & yes & yes & yes & yes & yes & no \\
\hline 9 & yes & yes & yes & yes & yes & yes & yes & no & no \\
\hline 10 & yes & yes & yes & yes & yes & yes & no & yes & no \\
\hline 11 & yes & yes & yes & yes & yes & yes & yes & yes & no \\
\hline 12 & yes & yes & yes & yes & yes & yes & no & no & no \\
\hline 13 & yes & yes & yes & yes & yes & yes & yes & yes & no \\
\hline 14 & no & yes & no & no & yes & yes & no & no & no \\
\hline 15 & yes & yes & no & yes & yes & yes & yes & yes & no \\
\hline 16 & no & yes & no & no & yes & yes & yes & no & no \\
\hline$n / N$ & $14 / 16$ & $16 / 16$ & $12 / 16$ & $11 / 16$ & $16 / 16$ & $16 / 16$ & $9 / 16$ & $10 / 16$ & $0 / 16$ \\
\hline$\%$ & 87.5 & 100 & 75 & 68.75 & 100 & 100 & 56.25 & 62.5 & 0 \\
\hline
\end{tabular}

PDRIS, premature desquamation of the inner root sheath; AGA, androgenetic alopecia. 
Fig. 1. a Cicatricial alopecia in a male androgenetic distribution. Bitemporal (arrow) and parietal hair loss. a1 Hyperpigmented perifollicular halo, scattered white small patches, hair diameter diversity, loss of follicular ostia. a2 AGA, horizontal section, papillary dermis with some terminal and many vellus hairs. b Cicatricial alopecia in a female androgenetic distribution. b1 Perifollicular erythema and scaling, loss of follicular ostia, keratotic follicular plugs, and hair diameter diversity. b2 Concentric fibrosis and fibrous tracts. c Cicatricial alopecia in a female androgenetic distribution. Arrow shows cicatricial parietal involvement. c1 Loss of follicular ostia, white perifollicular ostia, hair diameter diversity, and a honeycomb hyperpigmented network. c2 Concentric fibrosis with intense perifollicular lymphocytic infiltrate. Terminal and vellus hair involvement. d Cicatricial alopecia in a female androgenetic distribution with parietal involvement. d1 Loss of follicular ostia, perifollicular erythema and scaling, and hair diameter diversity. d2 AGA features with a lichenoid infiltrate around the vellus hair. Premature desquamation of the inner root sheath. e Diffuse hair thinning associated with recession of the frontotemporal hairline. Arrows showing the recession and the eyebrow thinning. e1 Perifollicular scaling, loss of follicular ostia, a white perifollicular halo and perifollicular scaling, and hair diameter diversity. e2 Concentric fibrosis with perifollicular lymphocytic infiltrate. $\mathbf{f}$ Cicatricial alopecia in a female androgenetic distribution with a central nonaffected parietal area (arrow). f1 Loss of follicular ostia, a whitish and hyperpigmented perifollicular halo, white patches, hair diameter diversity, and a honeycomb pigmented network. $\mathbf{f} 2$ Vellus hair involvement. g Diffuse hair thinning with a temporal nonaffected area. g1 Loss of follicular ostia, a whitish and hyperpigmented perifollicular halo, white patches, hair diameter diversity, and a honeycomb pigmented network. g2 Premature desquamation of the inner root sheath.
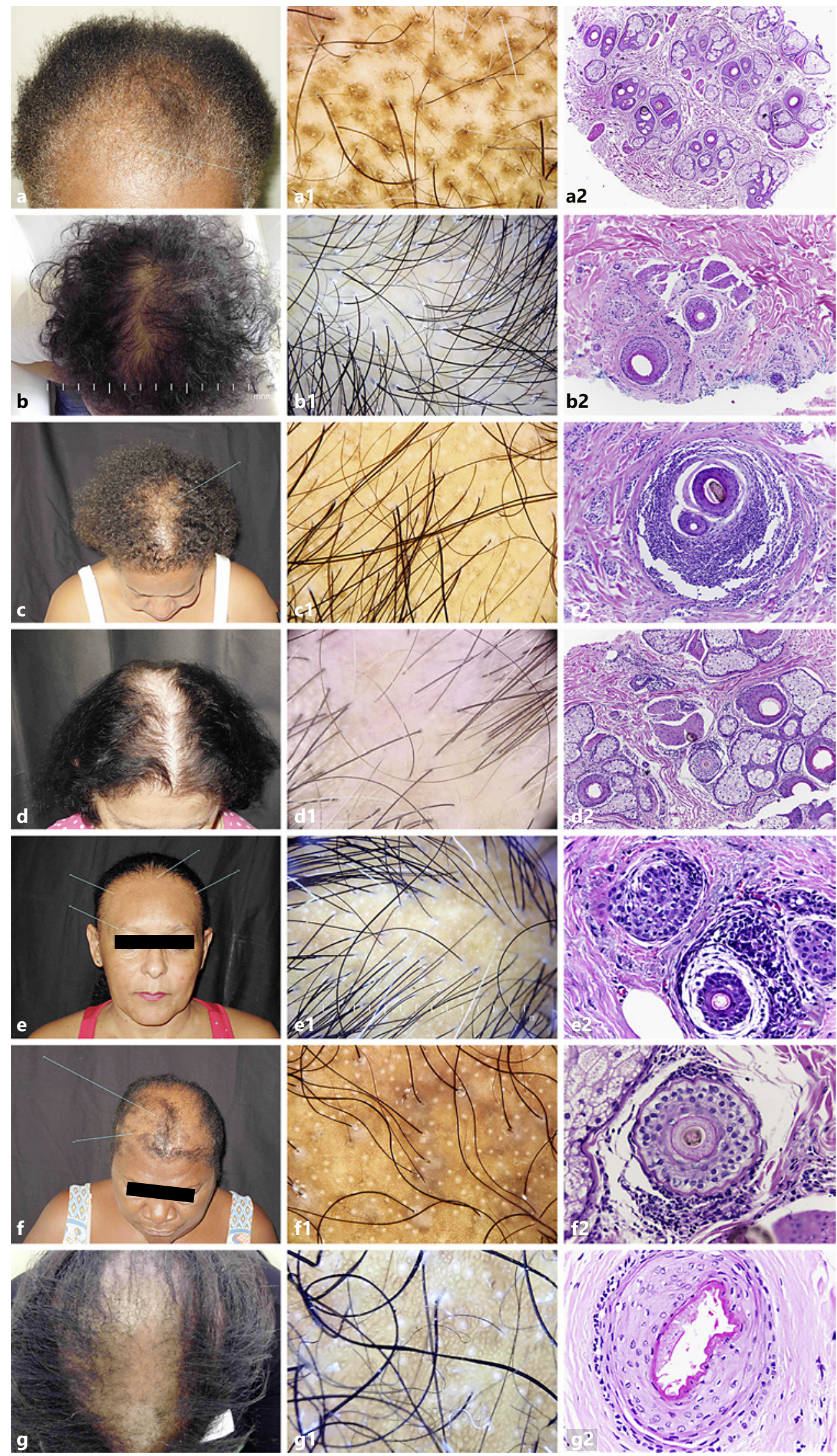

FAPD in African-Descent and Hispanic Female Patients

Skin Appendage Disord 2019;5:211-215 DOI: $10.1159 / 000496837$ 
[9-11]. The authors described patients with FAPD with clinical features of AGA [9] and cicatricial findings, some of them presenting with the involvement of the frontal hair line $[1,12,13]$. Since the original reports, evidence has accumulated that considerable clinical overlap exists between FFA and FAPD, or progression between these conditions, suggesting that FAPD may involve the frontal hairline $[1,3,4,12]$. A pure form of FFA does not present hair miniaturization associated with a lichenoid perifollicular infiltrate and concentric fibrosis in the same scalp biopsy, but the possibility of the existence of the two entities in the same patient must be considered $[2,4,14]$.

The anisotrichia typical of AGA and FAPD was described by Miteva and Tosti [7] in $94 \%$ of their samples with central centrifugal cicatricial alopecia (CCCA), compared to $100 \%$ of our patients. Other findings such as white patches, a perifollicular white halo, and a honeycomb pigmented network described by Miteva and Tosti [7] in patients with CCCA were also present in our patients with FAPD, concluding that dermatoscopy features of CCCA and FAPD in patients of color are very similar and may not be enough to establish the diagnosis.

While CCCA is a centrifugal expanding alopecia patch $[7,15]$, FAPD is a diffuse hair loss in a pattern distribution barely forming a real patch with hair diameter diversity as seen in AGA $[1,3,6]$. In FAPD, there is a continuous loss of hair density and hair follicle miniaturization clinically resembling AGA, with a lichenoid infiltrate or true fibrous tracts $[1,6]$. Premature desquamation of the inner root sheath [2] was found in $68,8 \%$ of the patients, suggesting that it may be present in patients of color with FAPD. The idea of multifactorial alopecia presented by Wohltmann and Sperling [16] is possible, but the interface alteration (lichenoid vacuolar-interface dermatitis) of the follicular epithelium is not found in CCCA [2].

The absence of an interface dermatitis targeting the vellus hair in 6 of the 16 patients does not exclude FAPD due to the difficulty of finding viable acute affected vellus hair in a small biopsy, especially in the late phases [2].

Until now, FAPD is not considered to exist in patients of color in whom CCCA is a mainstream diagnosis for mid-scalp cicatricial alopecia [17, 18]. Mardones et al. [4] were the first to describe the evidence of FAPD amongst Latin Americans in Chile. However, the Chilean population mostly consists of White and Amerindian people so that African patients were not particularly analyzed in that study.

We encourage the inclusion of FAPD as a possible diagnosis in patients of color who present mid-scalp cicatricial pattern alopecia. As CCCA is primarily described as a non-lichenoid lymphocytic cicatricial alopecia, as by Sperling et al. [2], we strongly suggest that the presence of histological features of AGA along with a lichenoid perifollicular lymphocytic infiltrate may allow the diagnosis of FAPD $[1,2]$.

\section{Conclusion}

FAPD is a possible diagnosis in patients of color who present mid-scalp pattern male or female cicatricial alopecia.

The concomitant finding of cicatricial pattern hair loss (with or without recess of the front hair line), hair fiber diversity, perifollicular erythema and scaling, a whitish perifollicular halo, histological findings of AGA, and vacuolar-interface alteration of the upper portion of the follicular epithelium are the main key features to suggest the diagnosis of FAPD. Due to the increasing understanding of trichoscopy, FAPD in patients of color may be suspected in early stages and be differentiated from CCCA, avoiding the progression of a permanent alopecia.

Some cases of FAPD might well include CCCA and FFA as a case of multifactorial alopecia, and a clinicaldermatoscopic-histopathological correlation is needed to establish the final diagnosis.

\section{Statement of Ethics}

Subjects have given their informed consent for participating in the study. The study protocol has been approved by the institute's committee on human research.

\section{Disclosure Statement}

The authors declare that they have no conflicts of interest to disclose.

References

1 Zinkernagel MS, Trüeb RM. Fibrosing alopecia in a pattern distribution: patterned lichen planopilaris or androgenetic alopecia with a lichenoid tissue reaction pattern? Arch Dermatol. 2000 Feb;136(2):205-11

2 Sperling LC, Cowper SE, Knopp EA. An atlas of hair pathology with clinical correlations. 2nd ed. New York and London: Informa healthcare; 2012

3 Missio DM, Dias MF, Trüeb RM. Familial Cicatricial Alopecia: Report of Familial Frontal Fibrosing Alopecia and Fibrosing Alopecia in a Pattern Distribution. Int J Trichology. 2017 Jul-Sep;9(3):130-4. 
4 Mardones F, Hott K, Martinez MC. Clinical study of fibrosing alopecia in a pattern distribution in a Latin American population. Int $\mathrm{J}$ Dermatol. 2018 Feb;57(2):e12-4.

5 Olsen EA. Female pattern hair loss and its relationship to permanent/cicatricial alopecia: a new perspective. J Investig Dermatol Symp Proc. 2005 Dec;10(3):217-21.

6 Chiu HY, Lin SJ. Fibrosing alopecia in a pattern distribution. J Eur Acad Dermatol Venereol. 2010 Sep;24(9):1113-4.

7 Miteva M, Tosti A. Dermatoscopic features of central centrifugal cicatricial alopecia. J Am Acad Dermatol. 2014 Sep;71(3):443-9.

8 Whiting DA. Diagnostic and predictive value of horizontal sections of scalp biopsy specimens in male pattern androgenetic alopecia. J Am Acad Dermatol. 1993 May;28(5 Pt 1): 755-63.
9 Hamilton JB. Patterned loss of hair in man; types and incidence. Ann N Y Acad Sci. 1951 Mar;53(3):708-28.

10 Pringle JJ (cited in Adamson HG). Lichen pilaris seu spinulosus. Br J Dermatol. 1905; 17(77).

11 Kossard S. Postmenopausal frontal fibrosing alopecia. Scarring alopecia in a pattern distribution. Arch Dermatol. 1994 Jun;130(6):7704.

12 Trémezaygues L, Vogt T, Müller CS. [Frontal fibrosing alopecia with androgenetic pattern. A diagnostic challenge - a therapeutic problem]. Hautarzt. 2012 May;63(5):411-4.

13 Moreno-Ramírez D, Camacho Martínez F. Frontal fibrosing alopecia: a survey in $16 \mathrm{pa}-$ tients. J Eur Acad Dermatol Venereol. 2005 Nov;19(6):700-5.
14 Rossi A, Grassi S, Fortuna MC, Garelli V, Pranteda G, Caro G, et al. Unusual patterns of presentation of frontal fibrosing alopecia: A clinical and trichoscopic analysis of $98 \mathrm{pa}-$ tients. J Am Acad Dermatol. 2017 Jul;77(1): 172-4.

15 LoPresti P, Papa CM, Kligman AM. Hot comb alopecia. Arch Dermatol. 1968 Sep;98(3): 234-8.

16 Wohltmann WE, Sperling L. Histopathologic diagnosis of multifactorial alopecia. J Cutan Pathol. 2016 Jun;43(6):483-91.

17 Sperling LC, Sau P. The follicular degeneration syndrome in black patients. 'Hot comb alopecia' revisited and revised. Arch Dermatol. 1992 Jan;128(1):68-74.

18 Miteva M, Tosti A. Pathologic diagnosis of central centrifugal cicatricial alopecia on horizontal sections. Am J Dermatopathol. 2014 Nov;36(11):859-64. 